\title{
Ricci tensor of Hopf hypersurfaces in a complex space form
}

\author{
Mayuko Kon* \\ (Communicated by Ramesh Sharma)
}

\begin{abstract}
We classify Hopf hypersurfaces in a non-flat complex space form whose Ricci tensor $S$ satisfies $g\left(\left(\nabla_{X} S\right) X, \xi\right)=0$ for any vector field $X$ tangent to $\xi$, where $\xi$ is the structure vector field. We also classify real hypersurfaces with transversal Killing Ricci tensor satisfying $S \xi=\beta \xi$ for some function $\beta$.
\end{abstract}

Keywords: Ricci tensor; real hypersurface; complex space form; transversal Killing tensor.

AMS Subject Classification (2010): Primary: 53B25 ; Secondary: 53C55; 53C25.

\section{Introduction}

For real hypersurfaces in a complex space form $M_{n}(4 c)$ of constant holomorphic sectional curvature $4 c \neq 0$, it is an interesting problem to determine real hypersurfaces satisfying an additional condition on the Ricci tensor.

Ki [3] showed that there are no real hypersurfaces with parallel Ricci tensor, $\nabla S=0$, in $M_{n}(4 c), n \geq 3$. Several conditions that weaken the condition $\nabla S=0$ are studied (cf., [4], [11]). On the other hand, when the structure vector field $\xi$ is principal, then the real hypersurface is said to be Hopf. For Hopf hypersurfaces, fundamental formulas are well-organized form, and it was considered to be a natural condition. So kinds of classification theorems are given under this assumption (see, for example, [10]). If the Ricci tensor $S$ satisfies $g\left(\left(\nabla_{X} S\right) Y, Z\right)=0$ for any vector field $X, Y$ and $Z$ orthogonal to $\xi$, then it is said to be $\eta$-parallel (Suh [11]). Suh and Maeda classified Hopf hypersurfaces of $M_{n}(4 c)$ with $\eta$-parallel Ricci tensor ([11], [9]). In [8], Maeda gave a classification of Hopf hypersurfaces in $\mathbb{C} P^{n}$ with $\nabla_{\xi} S=0$.

When $S$ satisfies $g\left(\left(\nabla_{X} S\right) X, \xi\right)=0$ for any $X$ orthogonal to $\xi$, we call $S$ the transversal $\eta$-Killing Ricci tensor. In section 3, we classify Hopf hypersurfaces whose Ricci tensor $S$ is transversal $\eta$-Killing.

In [6] and [7], the author showed that If $\left(\nabla_{X} S\right) Y$ is proportional (resp. perpendicular) to the structure vector field $\xi$ for any vector fields $X$ and $Y$ orthogonal to $\xi$, then $M$ is a Hopf hypersurface (resp. ruled real hypersurface), under an assumption that $S \xi=\beta \xi, \beta$ being a function. On the other hand, for an almost contact metric manifold $(M, \phi, \eta, \xi, g)$, Cho [2] considered a condition that a (1,1)-tensor field $T$ on $M$ a transversal Killing tensor field, that is, it satisfies $\left(\nabla_{X} T\right) X=0$ for any vector fields $X$ to $\xi$.

Combining these with the results in section 3, we classify real hypersurfaces of $M_{n}(4 c)$ whose Ricci tensor $S$ is a transversal Killing tensor field and satisfies $S \xi=\beta \xi$ for some function $\beta$, in section 4 . We notice that any Hopf hypersurfaces and ruled real hypersurfaces satisfy the condition that $S \xi=\beta \xi, \beta$ beging a function.

The author would like to express her sincere gratitude to the referee for valuable suggestions and comments.

\section{Preliminaries}

Let $M_{n}(4 c)$ denote the complex space from of complex dimension $n$ (real dimension $2 n$ ) of constant holomorphic sectional curvature $4 c$. For the sake of simplicity, if $c>0$, we only use $c=+1$ and call it the complex projective space $\mathbb{C} P^{n}$, and if $c<0$, we just consider $c=-1$, so that we call it the complex hyperbolic space $\mathbb{C} H^{n}$. Throughout this paper, we suppose that $c \neq 0$. We denote by $J$ the almost complex structure of $M_{n}(4 c)$. The Hermitian metric of $M_{n}(4 c)$ will be denoted by $G$. 
Let $M$ be a real $(2 n-1)$-dimensional hypersurface immersed in $M_{n}(4 c)$. We denote by $g$ the Riemannian metric induced on $M$ from $G$. We take the unit normal vector field $N$ of $M$ in $M_{n}(4 c)$. For any vector field $X$ tangent to $M$, we define $\phi, \eta$ and $\xi$ by

$$
J X=\phi X+\eta(X) N, \quad J N=-\xi
$$

where $\phi X$ is the tangential part of $J X, \phi$ is a tensor field of type (1,1), $\eta$ is a 1-form, and $\xi$ is the unit vector field on $M$. Then they satisfy

$$
\phi^{2} X=-X+\eta(X) \xi, \quad \phi \xi=0, \quad \eta(\phi X)=0
$$

for any vector field $X$ tangent to $M$. Moreover, we have

$$
\begin{aligned}
& g(\phi X, Y)+g(X, \phi Y)=0, \quad \eta(X)=g(X, \xi), \\
& g(\phi X, \phi Y)=g(X, Y)-\eta(X) \eta(Y) .
\end{aligned}
$$

Thus $(\phi, \xi, \eta, g)$ defines an almost contact metric structure on $M$.

We denote by $\tilde{\nabla}$ the operator of covariant differentiation in $M_{n}(4 c)$, and by $\nabla$ the one in $M$ determined by the induced metric. Then the Gauss and Weingarten formulas are given respectively by

$$
\tilde{\nabla}_{X} Y=\nabla_{X} Y+g(A X, Y) N, \quad \tilde{\nabla}_{X} N=-A X
$$

for any vector fields $X$ and $Y$ tangent to $M$. We call $A$ the shape operator of $M$. If the shape operator $A$ of $M$ satisfies $A \xi=\alpha \xi$ for some functions $\alpha$, then $M$ is said to be Hopf. We use the following (cf. [10])

Lemma 2.1. Let $M$ be a Hopf hypersurface of $M_{n}(4 c), n \geq 2, c \neq 0$. If a vector field $X$ is orthogonal to $\xi$ and $A X=\lambda X$, then

$$
(2 \lambda-\alpha) A \phi X=(\lambda \alpha+2 c) \phi X
$$

where $\alpha=g(A \xi, \xi)$, and $\alpha$ is constant.

For the almost contact metric structure on $M$, we have

$$
\nabla_{X} \xi=\phi A X, \quad\left(\nabla_{X} \phi\right) Y=\eta(Y) A X-g(A X, Y) \xi .
$$

We denote by $R$ the Riemannian curvature tensor field of $M$. Then the equation of Gauss is given by

$$
\begin{aligned}
R(X, Y) Z= & c\{g(Y, Z) X-g(X, Z) Y+g(\phi Y, Z) \phi X \\
& -g(\phi X, Z) \phi Y-2 g(\phi X, Y) \phi Z\} \\
& +g(A Y, Z) A X-g(A X, Z) A Y,
\end{aligned}
$$

and the equation of Codazzi by

$$
\left(\nabla_{X} A\right) Y-\left(\nabla_{Y} A\right) X=c\{\eta(X) \phi Y-\eta(Y) \phi X-2 g(\phi X, Y) \xi\} .
$$

From the equation of Gauss, the Ricci tensor $S$ of $M$ satisfies

$$
\begin{aligned}
g(S X, Y)= & (2 n+1) c g(X, Y)-3 c \eta(X) \eta(Y) \\
& +\operatorname{Tr} A g(A X, Y)-g(A X, A Y),
\end{aligned}
$$

where $\operatorname{Tr} A$ is the trace of $A$. By (2.1), we have

$$
\begin{aligned}
\left(\nabla_{X} S\right) Y= & -3 c g(\phi A X, Y) \xi-3 c \eta(Y) \phi A X \\
& +(X \operatorname{Tr} A) A Y+\operatorname{Tr} A\left(\nabla_{X} A\right) Y-A\left(\nabla_{X} A\right) Y \\
& -\left(\nabla_{X} A\right) A Y
\end{aligned}
$$

We use the following results to prove our theorem (see [1], [5], [10], [12], [13]).

Theorem A. Let $M$ be a real hypersurface of $M_{n}(4 c)$. Then the principal curvatures of $M$ are constant and $\xi$ is principal, if and only if, $M$ is an open subset of a homogeneous hypersurfaces.

Theorem B. Let $M$ be a homogeneous real hypersurface of $\mathbb{C} P^{n}$. Then $M$ is congruent to one of the following: 
$\left(A_{1}\right)$ a geodesic sphere of radius $r$, where $0<r<\pi / 2$,

$\left(A_{2}\right)$ a tube of radius $r$ over a totally geodesic $\mathbb{C} P^{k}(1 \leq k \leq n-2)$, where $0<r<\pi / 2$,

(B) A tube of radius $r$ around a complex hyperquadric $\mathbb{C} Q^{n-1}$, where $0<r<\pi / 4$.

(C) a tube of radius $r$ over $a \mathbb{C} P^{1} \times \mathbb{C} P^{\frac{n-1}{2}}$, where $0<r<\pi / 4$ and $n(\geq 5)$ is odd,

$(D)$ a tube of radius $r$ over a complex Grassmann $G_{2,5}(\mathbb{C})$, where $0<r<\pi / 4$ and $n=9$,

(E) a tube of radius $r$ over a Hermitian symmetric space $S O(10) / U(5)$, where $0<r<\pi / 4$ and $n=15$.

The principal curvatures are as follows.

\begin{tabular}{|c|c|c|c|c|}
\hline & $\left(A_{1}\right)$ & $\left(A_{2}\right)$ & $(B)$ & $(C, D, E)$ \\
\hline$\lambda_{1}$ & $\cot r$ & $\cot r$ & $\cot (r-\pi / 4)$ & $\cot (r-\pi / 4)$ \\
\hline$\lambda_{2}$ & & $-\tan r$ & $\cot (r+\pi / 4)$ & $\cot (r+\pi / 4)$ \\
\hline$\lambda_{3}$ & & & & $\cot r$ \\
\hline$\lambda_{4}$ & & & & $-\tan r$ \\
\hline$\alpha$ & $2 \cot (2 r)$ & $2 \cot (2 r)$ & $2 \cot (2 r)$ & $2 \cot (2 r)$ \\
\hline
\end{tabular}

The multiplicity $m(\mu)$ of each principal curvature $\mu$ of a homogeneous real hypersurface is as follows.

\begin{tabular}{|c|c|c|c|c|c|c|}
\hline & $\left(A_{1}\right)$ & $\left(A_{2}\right)$ & $(B)$ & $(C)$ & $(D)$ & $(E)$ \\
\hline$\lambda_{1}$ & $2 n-2$ & $2 n-2 k-2$ & $n-1$ & 2 & 4 & 6 \\
\hline$\lambda_{2}$ & & $2 k$ & $n-1$ & 2 & 4 & 6 \\
\hline$\lambda_{3}$ & & & & $n-3$ & 4 & 8 \\
\hline$\lambda_{4}$ & & & & $n-3$ & 4 & 8 \\
\hline$\alpha$ & 1 & 1 & 1 & 1 & 1 & 1 \\
\hline
\end{tabular}

Theorem C. Let $M$ be a Hopf hypersurface of $\mathbb{C} H^{n}, n \geq 2$. If all principal curvatures are constant, then $M$ is locally congruent to one of the following:

$\left(A_{0}\right)$ A horosphere,

$\left(A_{1,0}\right)$ A geodesic sphere of radius $r(0<r<\infty)$,

$\left(A_{1,1}\right)$ A tube of radius $r$ around a totally geodesic $\mathbb{C} H^{n-1}(c)$, where $0<r<\infty$,

$\left(A_{2}\right)$ A tube of radius $r$ around a totally geodesic $\mathbb{C} H^{l}(c)(1 \leq l \leq n-2)$, where $0<r<\infty$,

(B) A tube of radius $r$ around a totally real totally geodesic $\mathbb{R} H^{n}(c / 4)$, where $0<r<\infty$.

The principal curvatures of these real hypersurfaces are given as follows:

\begin{tabular}{|c|c|c|c|c|c|}
\hline & $\left(A_{0}\right)$ & $\left(A_{1,0}\right)$ & $\left(A_{1,1}\right)$ & $\left(A_{2}\right)$ & $(B)$ \\
\hline$\lambda_{1}$ & 1 & $\operatorname{coth} r$ & $\tanh r$ & $\operatorname{coth} r$ & $\operatorname{coth} r$ \\
\hline$\lambda_{2}$ & & & & $\tanh r$ & $\tanh r$ \\
\hline$\alpha$ & 2 & $2 \operatorname{coth}(2 r)$ & $2 \operatorname{coth}(2 r)$ & $2 \operatorname{coth}(2 r)$ & $2 \tanh (2 r)$ \\
\hline
\end{tabular}

\section{The covariant derivative of the Ricci tensor}

Let $M$ be Hopf hypersurface of a complex space form $M_{n}(4 c), c \neq 0$. Then the shape operator $A$ satisfies $A e_{i}=a_{i} e_{i}, 1 \leq i \leq 2 n-2$, with respect to a suitable orthonormal frame $\left\{e_{1}, \cdots, e_{2 n-2}, \xi\right\}$. We remark that if $A_{i} e_{i}=a_{i} e_{i}$, then

$$
\left(2 a_{i}-\alpha\right) A \phi e_{i}=\left(a_{i} \alpha+2 c\right) \phi e_{i},
$$

by Lemma 2.1. In the following, we put $A \phi e_{i}=\bar{a}_{i} \phi e_{i}$. Then we have

$$
2 a_{i} \overline{a_{i}}-a_{i} \alpha-\overline{a_{i}} \alpha-2 c=0 .
$$

Lemma 3.1. Let $M$ be a Hopf hypersurface of $M_{n}(4 c)$. The Ricci tensor $S$ of $M$ is transversal $\eta$-Killing if and only if

$$
\left(a_{i}-a_{j}\right)\left(-3 c+\alpha \operatorname{Tr} A-\alpha^{2}-a_{i} a_{j}\right) g\left(\phi e_{i}, e_{j}\right)=0
$$

for $i, j=1, \cdots, 2 n-2$. 
Proof. By (2.2), when $M$ is a Hopf hypersurface of $M_{n}(4 c)$, we obtain

$$
\begin{aligned}
& g\left(\left(\nabla_{e_{i}} S\right) e_{j}, \xi\right) \\
& =-3 c a_{i} g\left(\phi e_{i}, e_{j}\right)+\left(\operatorname{Tr} A-\alpha-a_{j}\right) g\left(\left(\nabla_{i} A\right) e_{j}, \xi\right) \\
& =-3 c a_{i} g\left(\phi e_{i}, e_{j}\right)+a_{i}\left(\operatorname{Tr} A-\alpha-a_{j}\right)\left(\alpha-a_{j}\right) g\left(\phi e_{i}, e_{j}\right) \\
& =a_{i}\left(-3 c+\alpha \operatorname{Tr} A-a_{j} \operatorname{Tr} A-\alpha^{2}+a_{j}^{2}\right) g\left(\phi e_{i}, e_{j}\right) .
\end{aligned}
$$

So we have

$$
\begin{aligned}
0 & =g\left(\left(\nabla_{e_{i}} S\right) e_{j}, \xi\right)+g\left(\left(\nabla_{e_{j}} S\right) e_{i}, \xi\right) \\
& =\left(a_{i}-a_{j}\right)\left(-3 c+\alpha \operatorname{Tr} A-\alpha^{2}-a_{i} a_{j}\right) g\left(\phi e_{i}, e_{j}\right) .
\end{aligned}
$$

First we suppose that $g\left(\left(\nabla_{X} S\right) X, \xi\right)=0$ for any $X$ orthogonal to $\xi$. Since $g\left(\left(\nabla_{X+Y} S\right)(X+Y), \xi\right)=0$ for any $X$ and $Y$ orthogonal to $\xi$, we have

$$
g\left(\left(\nabla_{X} S\right) Y, \xi\right)+g\left(\left(\nabla_{Y} S\right) X, \xi\right)=0 .
$$

So we have (3.3).

Next we suppose that the Ricci tensor $S$ satisfies (3.3). Then we obtain

$$
\begin{aligned}
& g\left(\left(\nabla_{e_{i}} S\right) e_{j}, \xi\right)+g\left(\left(\nabla_{e_{j}} S\right) e_{i}, \xi\right) \\
& =\left(a_{i}-a_{j}\right)\left(-3 c+\alpha \operatorname{Tr} A-\alpha^{2}-a_{i} a_{j}\right) g\left(\phi e_{i}, e_{j}\right)=0
\end{aligned}
$$

for any $i$ and $j$. Thus we get $g\left(\left(\nabla_{e_{i}} S\right) e_{i}, \xi\right)=0$. Any vector field $X$ orthogonal to $\xi$ is represented as $X=$ $\sum_{i} X_{i} e_{i}$. Using $g\left(\left(\nabla_{e_{i}} S\right) e_{j}, \xi\right)=-g\left(\left(\nabla_{e_{j}} S\right) e_{i}, \xi\right)$, we have

$$
\begin{aligned}
& g\left(\left(\nabla_{X} S\right) X, \xi\right) \\
& =\sum_{i, j} X_{i} X_{j} g\left(\left(\nabla_{e_{i}} S\right) e_{j}, \xi\right) \\
& =\sum_{i} X_{i}{ }^{2} g\left(\left(\nabla_{e_{i}} S\right) e_{i}, \xi\right)=0 .
\end{aligned}
$$

So we have our result.

Lemma 3.2. Let $M$ be a Hopf hypersurface of $M_{n}(4 c)$. If the Ricci tensor $S$ of $M$ is transversal $\eta$-Killing, then $M$ has at most 5 distinct constant principal curvatures.

Proof. From Lemma 3.1, putting $e_{j}=\phi e_{i}$ in (3.3), we have $a_{i}=\overline{a_{i}}$ or

$$
-3 c+\alpha \operatorname{Tr} A-\alpha^{2}-a_{i} \overline{a_{i}}=0 .
$$

If $a_{i}=\overline{a_{i}}$, by (3.2), we see that $a_{i}$ is a solution of the equation

$$
x^{2}-\alpha x-c=0 .
$$

Since $\alpha$ is constant, $a_{i}$ is also constant.

When $a_{i} \neq \bar{a}_{i}$, from (3.1), we have $2 a_{i}=\alpha$ or $\bar{a}_{i}=\frac{a_{i} \alpha+2 c}{2 a_{i}-\alpha}$. If $2 a_{i}=\alpha$ for some $a_{i}$, again from (3.1), we have $a_{i} \alpha+2 c=0$, from which we see that $\alpha^{2}=-4 c$ and $c<0$. Then $M$ has 2 constant principal curvatures (see [1]).

In the following, we suppose $2 a_{i} \neq \alpha$ for any $i$. From (3.4) and $\overline{a_{i}}=\frac{a_{i} \alpha+2 c}{2 a_{i}-\alpha}$, we see that $a_{i}$ is a solution of the following

$$
x^{2} \alpha-2\left(-4 c+\alpha \operatorname{Tr} A-\alpha^{2}\right) x+\alpha\left(-3 c+\alpha \operatorname{Tr} A-\alpha^{2}\right)=0 .
$$

We remark that $\overline{a_{i}}$ is also the solution of the above equation since (3.2) and (3.4) is symmetric with respect to $a_{i}$ and $\overline{a_{i}}$.

Therefore, we see that the shape operator $A$ has at most 5 distinct principal curvatures. We put $\lambda_{1}$ and $\lambda_{2}=\bar{\lambda}_{1}$ are solutions of (3.6), whose multiplicity is $k$, respectively. We suppose $\lambda_{3}, \lambda_{4}$ are solutions of (3.5) with multiplicity $l$ and $m$, respectively. Then we have

$$
\operatorname{Tr} A=k\left(\lambda_{1}+\overline{\lambda_{1}}\right)+l \lambda_{3}+m \lambda_{4}+\alpha .
$$


When $\alpha \neq 0$, since $\lambda_{1}$ and $\overline{\lambda_{1}}$ are solutions of (3.6), we have

$$
\lambda_{1}+\overline{\lambda_{1}}=\frac{2\left(-4 c+\alpha \operatorname{Tr} A-\alpha^{2}\right)}{\alpha} .
$$

From these equations, we obtain

$$
\alpha(1-2 k) \operatorname{Tr} A=\left(l \lambda_{3}+m \lambda_{4}\right) \alpha-8 k c-2 k \alpha^{2}+\alpha^{2} .
$$

Since $\alpha(1-2 k) \neq 0$, we see that $\operatorname{Tr} A$ is constant. By (3.6), $\lambda_{1}$ and $\overline{\lambda_{1}}$ are also constant. Hence all principal curvatures are constant.

Finally we consider the case that $\alpha=0$. If $a_{i} \neq \overline{a_{i}}$, then $a_{i}$ and $\overline{a_{i}}$ are solutions of (3.6). So we have $a_{i}=\overline{a_{i}}=0$. This is a contradiction. So we have $a_{i}=\overline{a_{i}}$ for all $a_{i}$. Then the principal curvatures are $\sqrt{c}$ and 0 with multiplicities $2 n-2$ and 1 , respectively.

Using these lemmas, we prove the following theorem.

Theorem 3.1. Let $M$ be a Hopf hypersurface of a complex projective space $\mathbb{C} P^{n}$. If the Ricci tensor $S$ of $M$ satisfies $g\left(\left(\nabla_{X} S\right) X, \xi\right)=0$ for any $X$ orthogonal to $\xi$, then $M$ is locally congruent to one of the following:

$\left(A_{1}\right)$ a geodesic sphere of radius $r$, where $0<r<\pi / 2$,

$\left(A_{2}\right)$ a tube of radius $r$ over a totally geodesic $\mathbb{C} P^{k}(1 \leq k \leq n-2)$, where $0<r<\pi / 2$,

(C) a tube of radius $r$ over a $\mathbb{C} P^{1} \times \mathbb{C} P^{\frac{n-1}{2}}$, where $\cot ^{2} 2 r=5 /(2 n-6)$ and $n(\geq 5)$ is odd,

$(D)$ a tube of radius $r$ over a complex Grassmann $G_{2,5}(\mathbb{C})$, where $\cot ^{2} 2 r=9 / 8$ and $n=9$,

(E) a tube of radius $r$ over a Hermitian symmetric space $S O(10) / U(5)$, where $\cot ^{2} 2 r=13 / 16$ and $n=15$.

Proof. By Lemma 3.2, when $M$ is a Hopf hypersurface in $\mathbb{C} P^{n}$ with at most 5 distinct principal curvatures. Therefore $M$ is locally congruent to one of the list in Theorem $\mathrm{B}$.

When $M$ is locally congruent to type $\left(A_{1}\right)$, then $\lambda_{1}=\cot r$ satisfies $\overline{\lambda_{1}}=\lambda_{1}$. Thus all principal curvatures satisfy (3.5). From Lemma 3.1, the Ricci operator $S$ of all type $\left(A_{1}\right)$ hypersurfaces satisfy $g\left(\left(\nabla_{X} S\right) X, \xi\right)=0$ for $X$ orthogonal to $\xi$. Similarly, since $\overline{\lambda_{1}}=\lambda_{1}$ and $\overline{\lambda_{2}}=\lambda_{2}$, type $\left(A_{2}\right)$ hypersurfaces also satisfy that condition.

Next we consider the case that $M$ is locally type $(B)$. The principal curvatures $\lambda_{1}=\cot \left(r-\frac{\pi}{4}\right)$ and $\lambda_{2}=$ $\cot \left(r+\frac{\pi}{4}\right)$ satisfies $\bar{\lambda}_{1}=\lambda_{2}$. If $\lambda_{1}$ and $\lambda_{2}$ are solutions of (3.6), then $\lambda_{1} \lambda_{2}=-3+\alpha \operatorname{Tr} A-\alpha^{2}=-1$. Since we have

$$
\operatorname{Tr} A=(n-1)\left(\cot \left(r-\frac{\pi}{4}\right)+\cot \left(r+\frac{\pi}{4}\right)\right)+\alpha,
$$

we see that

$$
\begin{aligned}
1 & =(n-1) \cot 2 r\left(\cot \left(r-\frac{\pi}{4}\right)+\cot \left(r+\frac{\pi}{4}\right)\right) \\
& =-2 n+2 .
\end{aligned}
$$

This is a contradiction. So type (B) hypersurfaces do not satisfy $g\left(\left(\nabla_{X} S\right) X, \xi\right)=0, X \perp \xi$.

Next we consider the case that $M$ has 5 distinct constant principal curvatures. We put

$$
\begin{aligned}
& \lambda_{1}=\cot \left(r-\frac{\pi}{4}\right), \lambda_{2}=\cot \left(r+\frac{\pi}{4}\right), \lambda_{3}=\cot r, \\
& \lambda_{4}=-\tan r, \alpha=2 \cot (2 r),
\end{aligned}
$$

and their multiplicities are represented by $m\left(\lambda_{1}\right)=m\left(\lambda_{2}\right)=k, m\left(\lambda_{3}\right)=m\left(\lambda_{4}\right)=l$. Since $\lambda_{1}$ and $\lambda_{2}$ are solutions of (3.6), similar computation as the case of type $(B)$ shows that $\operatorname{Tr} A \cdot \alpha-\alpha^{2}=2$. On the other hand, we obtain

$$
\begin{aligned}
\operatorname{Tr} A-\alpha & =k\left(\lambda_{1}+\lambda_{2}\right)+l\left(\lambda_{3}+\lambda_{4}\right) \\
& =\frac{4 k \tan ^{2} r-l\left(1-\tan ^{2} r\right)^{2}}{\left(\tan ^{2} r-1\right) \tan r} .
\end{aligned}
$$

Since $\alpha=2 \cot 2 r$, we have

$$
\alpha(\operatorname{Tr} A-\alpha)=-4 k+l\left(\frac{1-\tan ^{2} r}{\tan r}\right)^{2}=2,
$$


from which we see that

$$
\cot ^{2} 2 r=\frac{1+2 k}{2 l} .
$$

When $M$ is locally congruent to type $(C)$, then $k=2$ and $l=n-3$. Thus we have $\cot ^{2} 2 r=\frac{5}{2(n-3)}$. Next, when $M$ is locally congruent to $(D)$, we obtain $\cot ^{2} 2 r=\frac{9}{8}$. Finally, if $M$ is locally congruent to $(E)$, then we have $\cot ^{2} 2 r=\frac{13}{16}$.

Theorem 3.2. Let $M$ be a Hopf hypersurface of a complex hyperbolic space $\mathbb{C} H^{n}$. If the Ricci tensor $S$ of $M$ satisfies $g\left(\left(\nabla_{X} S\right) X, \xi\right)=0$ for any $X$ orthogonal to $\xi$, then $M$ is locally congruent to one of the following:

$\left(A_{0}\right)$ A horosphere,

$\left(A_{1,0}\right)$ A geodesic sphere of radius $r(0<r<\infty)$,

$\left(A_{1,1}\right)$ A tube of radius $r$ around a totally geodesic $\mathbb{C} H^{n-1}(c)$, where $0<r<\infty$,

$\left(A_{2}\right)$ A tube of radius $r$ around a totally geodesic $\mathbb{C} H^{l}(c)(1 \leq l \leq n-2)$, where $0<r<\infty$,

Proof. Similar argument as the proof of Theorem 3.1 shows that all type $\left(A_{0}\right),\left(A_{1,0}\right)$ and $\left(A_{1,1}\right)$ and $\left(A_{2}\right)$ hypersurface satisfies the condition $g\left(\left(\nabla_{X} S\right) X, \xi\right)=0$ for any $X$ orthogonal to $\xi$.

Suppose $M$ is locally congruent to type $(B)$. Then $\lambda_{1}=\operatorname{coth} r$ and $\lambda_{2}=\tanh r$ are solutions of (3.6). Then we have $\alpha \operatorname{Tr} A-\alpha^{2}=-2$. So we have

$$
\tanh 2 r(n-1)(\operatorname{coth} r+\tanh r)=-1 .
$$

By the straightforward computation, we have $2(n-1)=-1$. This is a contradiction.

\section{Transversal Killing tensor}

For a Riemannian manifold with Riemannian connection $\nabla$, a (1,1)-tensor field $T$ is called a Killing tensor field if it satisfies $\left(\nabla_{X} T\right) X=0$ or $\left(\nabla_{X} T\right) Y+\left(\nabla_{Y} T\right) X=0$ for any vector fields $X$ and $Y$. If $T$ is symmetric, then we easily see that $T$ is parallel. For an almost contact metric manifold $(M, \phi, \eta, \xi, g)$, we call a $(1,1)$ tensor field $T$ on $M$ a transversal Killing tensor field if it satisfies $\left(\nabla_{X} T\right) X=0$ or $\left(\nabla_{X} T\right) Y+\left(\nabla_{Y} T\right) X=0$ for any vector fields $X$ and $Y$ orthogonal to $\xi$ (see Cho[2]). Cho [2] studied a real hypersurfaces in a non-flat complex space form whose shape operator is a transversal Killing tensor field. In this section, we study a real hypersurface $M$ whose Ricci tensor $S$ is a transversal Killing tensor field. We summarize theorems for later use.

Theorem D ([7]). Let $M$ be a connected real hypersurface of $M_{n}(4 c), n \geq 3$, and suppose that the Ricci tensor $S$ of $M$ satisfies $S \xi=\beta \xi$ for some function $\beta$.

(1) If $\left(\nabla_{X} S\right) Y$ is proportional to the structure vector field $\xi$ for any vector fields $X$ and $Y$ orthogonal to $\xi$, then $M$ is a Hopf hypersurface.

(2) If $\left(\nabla_{X} S\right) Y$ is perpendicular to the structure vector field $\xi$ for any vector fields $X$ and $Y$ orthogonal to the structure vector field $\xi$, then $M$ is a ruled real hypersurface.

When $n=2$, the author gave a corresponding result in [6].

We use the following theorems for hypersurfaces with $\eta$-parallel Ricci tensor (see [9], [11]).

Theorem E. Let $M$ be a Hopf hypersurface of $\mathbb{C} P^{n}, n \geq 2$ with $\eta$-parallel Ricci tensor. Then $M$ is congruent to one of real hypersurfaces of types $\left(A_{1}\right),\left(A_{2}\right)$ and $(B)$ or a non-homogeneous real hypersurface with $A \xi=0$ in $\mathbb{C} P^{2}$.

Theorem F. Let $M$ be a Hopf hypersurface of $\mathbb{C} H^{n}, n \geq 2$ with $\eta$-parallel Ricci tensor. Then $M$ is congruent to one of real hypersurfaces of types $\left(A_{0}\right),\left(A_{1,0}\right),\left(A_{1,1}\right),\left(A_{2}\right)$ and $(B)$ or a non-homogeneous real hypersurface with $A \xi=0$ in $\mathbb{C} H^{2}$.

First, we prove the following lemma.

Lemma 4.1. Let $M$ be a connected real hypersurface of $M_{n}(4 c), n \geq 2$, and suppose that the Ricci tensor $S$ of $M$ is transversal Killing tensor field and satisfies $S \xi=\beta \xi$ for some function $\beta$, then $M$ is a Hopf hypersurface with $\eta$-parallel Ricci tensor. 
Proof. By the assumption we have $\left(\nabla_{X} S\right) X=0$ for any $X$ orthogonal to $\xi$, which is equivalent to $\left(\nabla_{X} S\right) Y+$ $\left(\nabla_{Y} S\right) X=0$ for any vector fields $X$ and $Y$ orthogonal to $\xi$. Since $S$ is symmetric, it follows that

$$
0=g\left(\left(\nabla_{X} S\right) X, Y\right)=-g\left(\left(\nabla_{Y} S\right) X, X\right) .
$$

This implies that $g\left(\left(\nabla_{X} S\right) Y, Z\right)=0$ for any vector fields $X, Y$ and $Z$ orthogonat to $\xi$. Hence, the Ricci tensor $S$ is $\eta$-parallel. Combining this to Theorem $\mathrm{D}(1), M$ is a Hopf hypersurface.

If the Ricci tensor $S$ of $M$ is transversal Killing tensor field, then $S$ is transversal $\eta$-Killing. Therefore, if a real hypersurface of $M_{2}(c)$ with $A \xi=0$ satisfies the condition that the Ricci tensor $S$ of $M$ is transversal Killing tensor field and $S \xi=\beta \xi$ for some function $\beta$, then Lemma 2.1 and Lemma 3.1 imply that $a_{1} a_{2}=c \neq 0$ and $\left(a_{1}-a_{2}\right)\left(a_{1} a_{2}-3 c\right)=0$. Thus $M$ is a totally $\eta$-umbilical real hypesurface. Thus a non-homogeneous real hypersurface with $A \xi=0$ in $M_{n}(4 c)$ does not satisfy the condition that the Ricci tensor $S$ of $M$ is transversal Killing tensor field and $S \xi=\beta \xi$ for some function $\beta$.

From Theorems 3.1, 3.2 we also see that real hypersurfaces of type (B) do not satisfy the condition that $S$ is transversal Killing tensor field and $S \xi=\beta \xi$ for some function $\beta$. Therefore we have the following theorems.

Theorem 4.1. Let $M$ be a real hypersurface of $\mathbb{C} P^{n}, n \geq 2$. If the Ricci tensor $S$ of $M$ is transversal Killing tensor field and satisfies $S \xi=\beta \xi$ for some function $\beta$, then $M$ is locally congruent to one of the types $\left(A_{1}\right)$ and $\left(A_{2}\right)$.

Theorem 4.2. Let $M$ be a real hypersurface of $\mathbb{C} H^{n}, n \geq 2$. If the Ricci tensor $S$ of $M$ is transversal Killing tensor field and satisfies $S \xi=\beta \xi$ for some function $\beta$, then $M$ is locally congruent to one of the types $\left(A_{0}\right),\left(A_{1,0}\right),\left(A_{1,1}\right)$ and $\left(A_{2}\right)$.

\section{References}

[1] Berndt, J., Real hypesurfaces with constant principal curvatures in comples hyperbolic space, J. Reine Angew. Math. 395 (2003), 11-25.

[2] Cho, J. T., Notes on real hypersurfaces in a complex space form, Bull. Korean Math. Soc. 52 (2015), 335-344.

[3] Ki, U.-H., Real hypersurfaces with parallel Ricci tensor of a complex space form, Tsukuba J. Math. 13 (1989), $73-81$.

[4] Ki, U-H., Nakagawa, H. and Suh, Y. J., Real hypersurfaces with harmonic Weyl tensor of a complex space form, Hiroshima Math. J. 20 (1990), 93-102.

[5] Kimura, M., Real hypersurfaces and complex submanifolds in complex projective space, Trans. Am. Math. Soc. 296 (1986), 137-149.

[6] Kon, M., 3-dimensional real hypersurfaces and Ricci operator, Diff. Geom. Dyn. Syst. 16 (2014), 189-202.

[7] Kon, M., Ricci tensor of real hypersurfaces, Pacific J. Math. 281 (2016), 103-123.

[8] Maeda, S., Ricci tensors of real hypersurfaces in a complex projective space, Proc. Amer. Math. Soc. 122 (1994), 1229-1235.

[9] Maeda, S., Hopf hypersurfaces with $\eta$-parallel Ricci tensors in a nonflat complex space forms, Scientiae Mathematicae Japonicae, 76 (2013), 449-456.

[10] Niebergall, R. and Ryan, P. J., Real hypersurfaces in complex space forms. Tight and taut submanifolds, Tight and taut submanifolds, 32 (1997), 233-305.

[11] Suh, Y. J., On real hypersurfaces of a complex space form with $\eta$-parallel Ricci tensor, Tsukuba J. Math. 14 (1990), 27-37.

[12] Takagi, R., Real hypersurfaces in a complex projective space with constant principal curvatures, J. Math. Soc. Japan 27 (1975), $43-53$.

[13] Takagi, R., Real hypersurfaces in a complex projective space with constant principal curvatures II, J. Math. Soc. Japan 27 (1975), $508-516$.

\section{Affiliations}

MAYUKO KON

ADDRESS: Faculty of Education, Shinshu University, 6-Ro, Nishinagano, Nagano City 380-8544, Japan

E-MAIL: mayuko_k@shinshu-u.ac.jp

ORCID ID : 0000-0002-2214-4238 